\title{
Prognostic Factors of Successful Cervical Ripening and Labor Induction in Late-Onset Fetal Growth Restriction
}

\author{
Cecilia Villalain María Soledad Quezada Paula Gómez-Arriaga Elisa Simón \\ Enery Gómez-Montes Alberto Galindo Ignacio Herraiz
}

Fetal Medicine Unit, Maternal and Child Health and Development Network (Red SAMID-RD12/0026/0016), Department of Obstetrics and Gynecology, University Hospital 12 de Octubre, 12 de Octubre Research Institute (imas12), Complutense University of Madrid, Madrid, Spain

\section{Keywords}

Labor induction - Fetal growth restriction - Cerebroplacental ratio · Foley balloon

\begin{abstract}
Objective: The aim of this work was to identify independent risk factors influencing the achievement of vaginal delivery among women undergoing labor induction for late-onset fetal growth restriction (FGR). Methods: This was a retrospective cohort study of 201 singleton pregnancies with late-onset FGR (diagnosed $>32+0$ weeks) that required labor induction with cervical ripening from $37+0$ weeks, either with dinoprostone (from 2014 to 2015) or Foley balloon (from 2016 to 2018). Independent factors for successful vaginal delivery were identified. A prediction model of vaginal delivery with the identified factors was made using logistic regression and bootstrapping with 1,000 re-samples performed for bias correction. Results: Perinatal results were more favorable in the vaginal delivery group, with significantly lower neonatal admission rates (4.0 vs. $13.7 \%)$ and lower composite neonatal morbidity ( 4.0 vs. $15.7 \%)$. The labor induction method (Foley balloon), higher cerebro-pla-
\end{abstract}

(c) 2019 S. Karger AG, Base

www.karger.com/fd

Karger" cental ratio, lower pre-gestational BMI, and absence of preeclampsia were identified as independent factors associated to vaginal delivery. The area under the curve of the model was of 0.75 (95\% Cl $0.70-0.79)$. Conclusions: The use of a Foley balloon is the only modifiable risk factor to improve the chances of vaginal delivery when attempting induction of labor in singleton pregnancies with late-onset FGR.

(c) 2019 S. Karger AG, Basel

\section{Introduction}

Fetal growth restriction (FGR) refers to an entity in which a fetus is not able to fully reach its growth potential. Excluding congenital anomalies, it is usually a consequence of a mismatch between fetal nutritional needs and placental supply. It is associated with a higher rate of perinatal and long-term morbidity and mortality [1]. Lateonset FGR refers to those diagnosed beyond 32 weeks [2] and affects $3-5 \%$ of pregnancies in our setting [3]. Although the moment during the slowing of the fetal growth curve in which prenatal identification of FGR occurs may fluctuate depending on the prenatal follow-up schedule, 
many of these late-identified cases are characterized by mild and stable hemodynamic alterations that allow, with close surveillance, prolonging the pregnancy until term $[4,5]$. Given that the risk of stillbirth for these fetuses increases from 37 weeks, there is a consensus that labor induction should be attempted once the early term period is reached [6].

The purpose of labor induction is to achieve vaginal delivery safely for both the mother and the fetus [7]. There is still controversy regarding the relationship between labor induction requiring cervical ripening and whether there is an increased risk or not of cesarean section (CS) $[8,9]$. In the particular setting of FGR, it is important to take into account that expectant management until the spontaneous onset of labor may not be an option [10]. There is scarce evidence on which is the best approach for cervical ripening in this scenario. Recently, our group has found that the Foley balloon could be an optimal method due to its lower association with uterine tachysystole when compared to prostaglandins [11]. Nevertheless, we hypothesize that, besides the method used, there may be other factors affecting the outcome of the induction process in this particular group of fetuses. The aim of this study was to determine which factors play an independent role in the success of labor induction in lateonset FGR and to elaborate a predictive model for vaginal delivery.

\section{Materials and Methods}

We present a retrospective cohort study on 370 consecutive gestations with late-onset (diagnosed $\geq 32$ weeks) stage I FGR attending our center between January 2014 and October 2018. We considered stage I FGR [12] as: those fetuses with an estimated fetal weight $(\mathrm{EFW})<\mathrm{p} 3$ and normal maternal and fetal Doppler; EFW $<$ p10, mean uterine arteries (mUtA) pulsatility index (PI) $>$ p95, and normal fetal Doppler; EFW $<$ p10 with mild fetal Doppler alterations, including umbilical artery (UA) PI $>95$ th centile, or middle cerebral artery (MCA) PI $<5$ th centile, or cerebro-placental ratio $(\mathrm{CPR})<5$ th centile.

Exclusion criteria were multiple pregnancies $(n=23)$, major congenital malformations $(n=14)$, fetal chromosomal anomalies $(n=2)$, or loss to follow-up $(n=5)$, resulting in 326 singleton gestations with late-onset FGR of suspected placental origin. Of them, we selected those that required labor induction at term $(\geq 37+0$ weeks) with cervical ripening for a Bishop score $<6(n=242)$. Regarding the cervical ripening method used, between January 2014 and December 2015, vaginal dinoprostone $10 \mathrm{mg}$ (Propess ${ }^{\circledR}$, Ferring Pharmaceutical, Saint-Prex, Switzerland) was used. In December 2015, a change in the cervical ripening method was introduced in our hospital according to expert recommendations that proposed that mechanical methods could be beneficial given its association with lower uterine tachysystole rates [13]. In these cas- es, labor induction was initiated with a Foley balloon filled with 30 $\mathrm{mL}$ of sterile water and traction was applied every $3 \mathrm{~h}$. Those cases that did not receive the expected cervical ripening method were further excluded, resulting in 201 cases ( $n=81$ in the vaginal dinoprostone group, and $n=120$ in the Foley balloon group) included for analysis. A flow chart summarizing the selection process and the method used for cervical ripening is depicted in Figure 1. The study was approved by our hospital's Ethics Committee, and the collection of informed consent was not required due to its retrospective character and the use of encoded and anonymized data.

During the study period, all women with suspicion of a small for gestational age fetus at the third trimester growth scan performed between 34 and 36 weeks or by palpation of the uterine fundal height were referred to the Fetal Medicine Unit for evaluation. A new morphological evaluation and fetal biometry were performed, and the study was completed with a maternal and fetal Doppler evaluation. The maternal Doppler evaluation included the study of the mUtA PI and the fetal Doppler evaluation, the UA PI, MCA PI, and CPR; if any of the fetal Doppler parameters were altered, the study was completed by adding an evaluation of the ductus venous. Fetal weight estimation was calculated using Hadlock's formula and customized according to ethnicity, age, parity, and pre-gestational BMI using the software GROW - Gestation Related Optimal Weight (available at http://www.gestation.net/ cc/6/884259.htm). The EFW used for analysis was the one obtained on the last scan, always performed on the same week of labor induction. All FGR cases, independently of their sonographic characteristics, were followed-up with weekly Doppler ultrasound and a non-stress test until $37+0$ weeks, recommending the induction of labor thenceforth. Those in which the diagnosis was made after term were delivered soon after.

According to our labor induction protocol, both methods were maintained for a maximum of $24 \mathrm{~h}$ ( $12 \mathrm{~h}$ for those with a prior CS) with continuous cardiotocographic monitoring, re-evaluating cervical dilation every $6 \mathrm{~h}$. Once a Bishop score of $\geq 7$ was achieved, a spontaneous expulsion of the Foley balloon was detected, or the maximum time for cervical ripening achieved, and after $30 \mathrm{~min}$ of reassuring cardiotocography (CTG), artificial rupture of membranes was performed. When necessary to regulate contractions, intravenous oxytocin was initiated at an infusion flow rate of 3 $\mathrm{mIU} / \mathrm{min}$ and increased periodically until 4-5 regular contractions per $10 \mathrm{~min}$ were achieved in the absence of CTG abnormalities. Nulliparous women who did not achieve cervical dilatation $>4 \mathrm{~cm}$ in the presence of adequate uterine contractions and despite $18 \mathrm{~h}$ of oxytocin augmentation, or $12 \mathrm{~h}$ in the case of multiparous women or those who had a previous CS, underwent CS for failure of labor induction.

If uterine tachysystole with fetal repercussion was identified, the cervical ripening method was withdrawn or the oxytocin perfusion interrupted. Fetal repercussion was considered as suspicious or pathological categories according to FIGO [14]. Once the fetal status recovered and after $30 \mathrm{~min}$ of reassuring CTG, the oxytocin perfusion was re-initiated.

The main variable in the study was the mode of delivery, differentiating neonates between those born vaginally and those delivered through CS independently of the indication for it (failed labor induction, labor arrest or cephalo-pelvic disproportion, malpresentation and non-reassuring fetal status [considered as a pathological CTG or a persistent one after resuscitation maneuvers according to the FIGO classification or fetal scalp sampling $\mathrm{pH} \leq 7.2]$ ). 


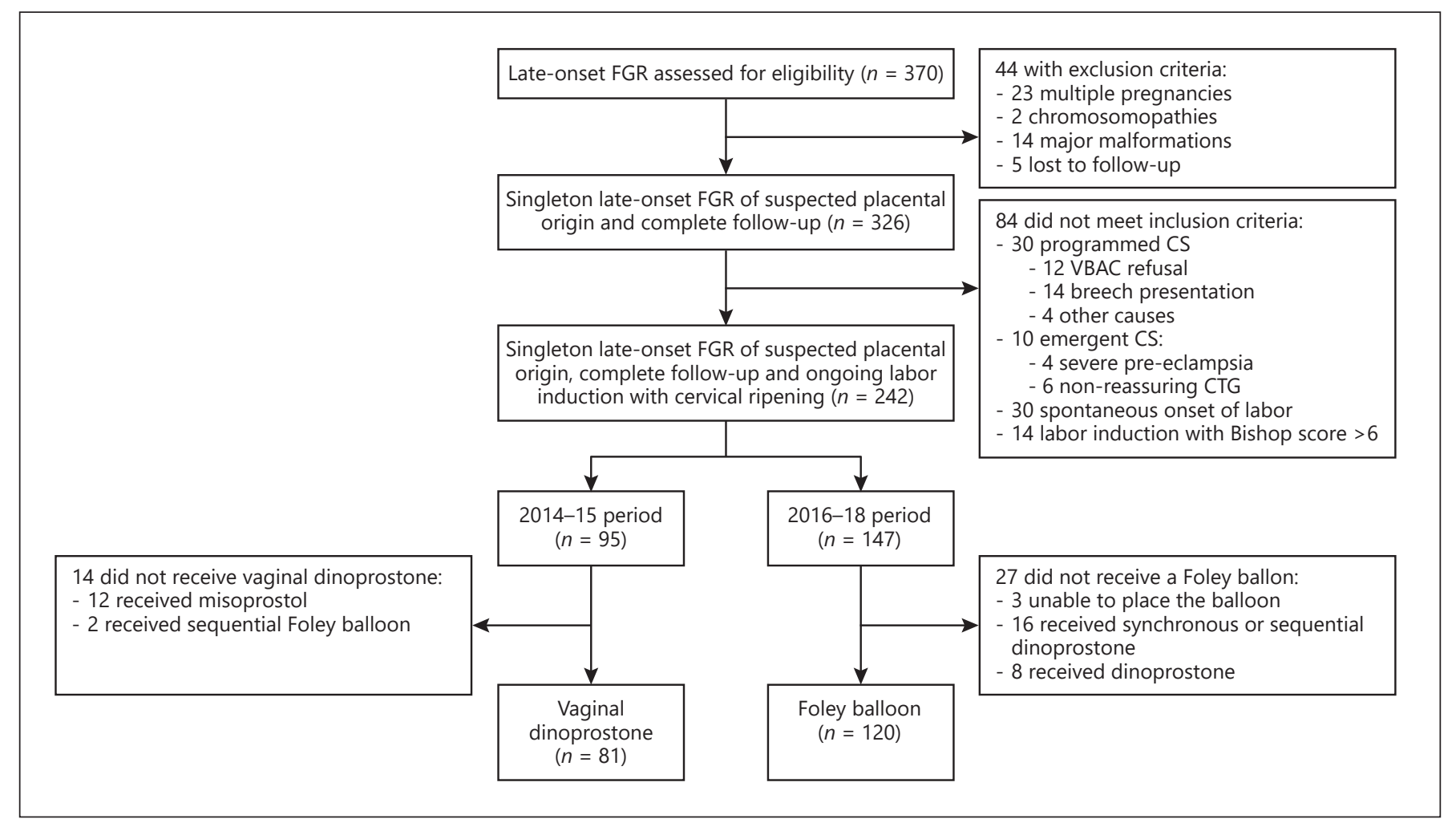

Fig. 1. Flow chart for the selection of the study population.

As independent variables, the main demographic characteristics such as maternal age, ethnicity, maternal height, weight, pregestational BMI, parity, gestational age at diagnosis of FGR and delivery, and Bishop score at admission were evaluated. We also took into consideration other possible maternal (pre-eclampsia [15] or pre-gestational diabetes), or induction (method for cervical ripening) conditioning factors.

Regarding perinatal results, we evaluated the arterial fetal $\mathrm{pH}$ at birth, Apgar score at $5 \mathrm{~min}$, neonatal intensive care unit (NICU) admission, mortality or severe neonatal morbidity (bronchopulmonary dysplasia, necrotizing enterocolitis, grade III or IV intraventricular hemorrhage, hypoxic-ischemic encephalopathy, or periventricular leukomalacia), and mortality and severe maternal morbidity. In order to better evaluate these rare events, we added the variable "composite neonatal morbidity" which included any of the parameters previously defined as severe neonatal morbidity, arterial $\mathrm{pH}<7$, Apgar score at $5 \mathrm{~min}$ $<7$, or NICU admission.

Aiming to elaborate a predictive model for vaginal delivery, we firstly conducted a descriptive analysis of the basal characteristics according to the mode of delivery (vaginal vs. CS), presenting quantitative data as the mean (SD) or median (range) according to the variable distribution and as count (percentage) if it was a qualitative variable. We performed a univariate analysis using parametric tests ( $t$ test, ANOVA, $\chi^{2}$ ) as needed.

To develop a prediction model, we firstly applied a non-linear transformation to each variable using the weight of evidence, calculated with the statistical machine-learning package scikit-learn v.0.20.2 in Python. For each variable a decision tree against the target variable is fitted. The parameters of the decision tree are chosen to avoid overfitting, with a maximum of 4 leaf nodes and a minimum of 10 samples per node. The splitting criterion used was the Gini inequality index [16]. We then calculated the target probability by applying the tree to each observation and computed the weight of evidence with the formula $\log ([1-p] / p)$. In order to avoid infinity values, probability was clipped between 0.001 and 0.999 . The weight of evidence-transformed variables were then used as input variables to a logistic regression.

We conducted a stepwise-backward variable selection method, including all items with a $p$ value $<0.20$ on the univariable analysis, retaining in the final model those with a $p$ value $<0.05$. In the iterative process of variable selection, variables were eliminated from the model if they were not statistically significant and non-confounders. In this case, the alpha error was established in 0.05 and any parameter that modified the estimation in $15 \%$ in relation to the complete model was considered a confounder.

The logistic regression model was performed using the transformed variables selected from the stepwise-backwards analysis. Associations were summarized using odds ratios and corresponding 95\% CIs estimated from the final multivariable model.

Finally, we evaluated the area under the curve (AUC) to assess its predictive power. An optimism-corrected, nearly unbiased estimate of the AUC estimate was derived using 1,000 bootstrap resamples as a method of internal validation. Statistical analysis was performed with the software STATA version 14.2. 
Table 1. Prenatal characteristics according to the mode of delivery

\begin{tabular}{|c|c|c|c|}
\hline & $\begin{array}{l}\text { Vaginal delivery } \\
(n=150)\end{array}$ & $\begin{array}{l}\mathrm{CS} \\
(n=51)\end{array}$ & $p$ value \\
\hline Maternal age, years & $32.1 \pm 6.2$ & $32.1 \pm 6.8$ & 0.49 \\
\hline \multicolumn{4}{|l|}{ Race/ethnicity } \\
\hline White & $104(69.3)$ & $41(80.4)$ & \multirow{5}{*}{0.33} \\
\hline Asian & $3(2.0)$ & $0(0.0)$ & \\
\hline North African & $12(8.0)$ & $4(7.8)$ & \\
\hline Hispanic & $30(20.0)$ & $5(9.8)$ & \\
\hline Black & $1(0.7)$ & $1(2.0)$ & \\
\hline BMI & $24.2 \pm 4.5$ & $26.4 \pm 6.2$ & $<0.01$ \\
\hline Height, cm & $162 \pm 7$ & $160 \pm 7$ & 0.96 \\
\hline Weight, kg & $63.8 \pm 12.0$ & $67.7 \pm 14.8$ & 0.03 \\
\hline Spontaneous conception & $142(94.7)$ & $13(61.5)$ & 0.26 \\
\hline Nulliparous & $100(68.0)$ & $47(92.2)$ & 0.18 \\
\hline Prior CS & $9(6.0)$ & $8(15.7)$ & 0.03 \\
\hline Pre-eclampsia & $13(8.7)$ & $17(33.3)$ & $<0.01$ \\
\hline Pre-gestational diabetes & $1(0.7)$ & $0(0)$ & 0.96 \\
\hline Smoker & $33(22.0)$ & $9(17.6)$ & 0.37 \\
\hline GA at diagnosis, weeks & $36.3 \pm 2.2$ & $35.9 \pm 2.2$ & 0.90 \\
\hline GA at delivery, weeks & $38.0 \pm 0.1$ & $37.4 \pm 1.6$ & 0.99 \\
\hline \multicolumn{4}{|l|}{$\mathrm{EFW}^{1}$} \\
\hline Grams & $2,248 \pm 282$ & $2,154 \pm 284$ & 0.96 \\
\hline Centile & $2[5]$ & $2[5]$ & 0.91 \\
\hline$<3$ rd centile & $98(65.3)$ & $33(64.7)$ & 0.89 \\
\hline \multicolumn{4}{|l|}{ UA Doppler } \\
\hline PI & $0.87 \pm 0.40$ & $0.91 \pm 0.35$ & 0.26 \\
\hline Centile & $69[70]$ & $94[70]$ & 0.48 \\
\hline$>95$ th centile & $56(37.3)$ & $25(49.0)$ & 0.18 \\
\hline \multicolumn{4}{|l|}{ UA } \\
\hline PI & $1.05 \pm 0.52$ & $1.20 \pm 0.29$ & $<0.01$ \\
\hline Centile & $60[38]$ & $40[30]$ & 0.02 \\
\hline$>95$ th centile & $17(11.3)$ & $15(29.4)$ & $<0.01$ \\
\hline \multicolumn{4}{|l|}{ MCA } \\
\hline PI & $1.50 \pm 0.35$ & $1.39 \pm 0.25$ & 0.02 \\
\hline Centile & $29[50]$ & $20[36]$ & 0.15 \\
\hline$<5$ th centile & $23(15.3)$ & $12(23.5)$ & 0.14 \\
\hline \multicolumn{4}{|l|}{$\mathrm{CPR}$} \\
\hline Mean \pm SD & $1.50 \pm 0.52$ & $1.21 \pm 0.35$ & $<0.01$ \\
\hline Centile & $10[32]$ & $3[9]$ & $<0.01$ \\
\hline$<5$ th centile & $65(43.3)$ & $36(70.6)$ & $<0.01$ \\
\hline \multicolumn{4}{|l|}{ Bishop score at admission } \\
\hline Median & $3[3]$ & $4[2]$ & 0.24 \\
\hline$<2$ & $40(26.7)$ & $19(37.3)$ & 0.15 \\
\hline \multicolumn{4}{|l|}{ Labor induction method } \\
\hline Foley balloon & $100(66.7)$ & $20(39.2)$ & $<0.01$ \\
\hline Dinoprostone & $50(33.3)$ & $31(60.8)$ & \\
\hline
\end{tabular}

Data are presented as the mean $\pm \mathrm{SD}, n(\%)$, or median [IQR]. CS, cesarean section; EFW, estimated fetal weight; UA, umbilical artery; MCA, middle cerebral artery; CPR, cerebro-placental ratio; NICU, neonatal intensive care unit; PI, pulsatility index; SD, standard deviation. ${ }^{1}$ Obtained at the last scan, performed within 7 days of delivery.

\section{Results}

The main basal maternal and fetal characteristics according to mode of delivery are shown in Table 1. Re- garding maternal characteristics, we observed lower $\mathrm{BMI}$, pre-gestational weight, pre-eclampsia incidence, and prior CS rates, as well as a higher proportion of Foley balloon use in the vaginal delivery group when com- 
Table 2. Perinatal results according to the mode of delivery

\begin{tabular}{lccc}
\hline & $\begin{array}{l}\text { Vaginal delivery } \\
(n=150)\end{array}$ & $\begin{array}{l}\text { CS } \\
(n=51)\end{array}$ & $p$ value \\
\hline Neonatal weight, $g$ & $2,340 \pm 327$ & $2,174 \pm 342$ & 0.08 \\
Arterial pH & $7.25 \pm 0.1$ & $7.20 \pm 0.1$ & 0.04 \\
Arterial pH $<7$ & $0(0)$ & $2(4.0)$ & 0.02 \\
Apgar at 5 min & $10[1]$ & $9[2]$ & 0.96 \\
Apgar at 5 min $<7$ & $6(4.0)$ & $3(6.1)$ & 0.54 \\
Severe morbidity & $0(0)$ & $7(0)$ & NA \\
NICU admission & $6(4.0)$ & $1(0-3)$ & 0.01 \\
Days at NICU & $0(0-2)$ & $8(15.7)$ & 0.54 \\
Composite neonatal morbidity & $6(4.0)$ & $<0.01$ \\
\hline
\end{tabular}

Data are presented as the mean $\pm \mathrm{SD}, n(\%)$, median $[\mathrm{IQR}]$, or median (range). CS, cesarean section; NICU, neonatal intensive care unit. ${ }^{1}$ Includes bronchopulmonary dysplasia, necrotizing enterocolitis, grade III or IV intraventricular hemorrhage, hypoxic-ischemic encephalopathy, or periventricular leukomalacia. ${ }^{2}$ Includes any of the parameters previously defined as severe neonatal morbidity, arterial $\mathrm{pH}<7$, Apgar score at 5 min $<7$, or NICU admission.

Table 3. Prediction model for vaginal delivery

\begin{tabular}{llllll}
\hline & Coefficient & SD & $z$ & $p>|z|$ & $95 \%$ CI \\
\hline Constant & -3.59 & 0.77 & -4.68 & $<0.001$ & -5.09 to -2.08 \\
Labor induction method: Foley & -1.3 & 0.36 & -3.66 & $<0.001$ & -2.00 to -0.60 \\
CPR centile & -1.12 & 0.30 & -3.73 & $<0.001$ & -1.71 to -0.53 \\
BMI & -1.05 & 0.41 & -2.54 & 0.011 & -1.85 to 0.24 \\
Pre-eclampsia & -0.84 & 0.29 & -2.91 & 0.004 & -1.41 to -0.28 \\
\hline
\end{tabular}

BMI, body mass index; CI, confidence interval; CPR, cerebro-placental ratio; SD, standard deviation.

pared to the CS group. There were no differences in the Bishop score at admission. Withdrawal of the cervical ripening method due to a non-reassuring fetal status was needed in 17 (8.5\%) cases. In the CS group, the indications for CS were a non-reassuring fetal status $(n=34$, $66.7 \%)$, failure of labor induction $(n=10,19.6 \%)$, labor arrest or cephalo-pelvic disproportion $(n=5,9.8 \%)$, and malpresentation diagnosed during labor $(n=2,3.9 \%)$. Regarding fetal Doppler, we found an overall lower rate of alterations in the vaginal delivery group (30.0 vs. $53.7 \%, p<0.01$ ), and in all individual MCA, UA, and CPR measurements.

There were no significant differences regarding basal patient characteristics between the period we were using dinoprostone and the period of Foley balloon use, except for gestational age at FGR diagnosis, which was slightly lower with the latter ( 36.4 vs. 35.8 weeks, $p=0.04$ ). Tachysystole with fetal repercussion was significantly more fre- quent in cases that used dinoprostone than in those with a Foley balloon ( 13.5 vs. $5.4 \%, p<0.05)$. There were also differences regarding the reason for indication of CS between both periods with a significantly higher rate of CS for non-reassuring fetal status within the dinoprostone period $(27.1$ vs. $8.3 \%, p<0.01)$. The perinatal results are presented in Table 2, with better results in the vaginal delivery group, which showed a higher arterial $\mathrm{pH}$ (7.25 vs. $7.20, p=0.04$ ), lower NICU admission rates (4.0 vs. $13.7 \%$, $p=0.01)$, and lower composite neonatal morbidity (4.0 vs. $15.7 \%, p=0.01$ ).

Finally, after transforming all variables to their weight of evidence, a multivariate model was elaborated to predict vaginal delivery in labor induction. As shown in Table 3 , the main predictive variables of successful vaginal delivery were the use of the Foley balloon, higher CPR centiles, lower pre-gestational BMI, and absence of preeclampsia. The vaginal delivery probability for each pre- 

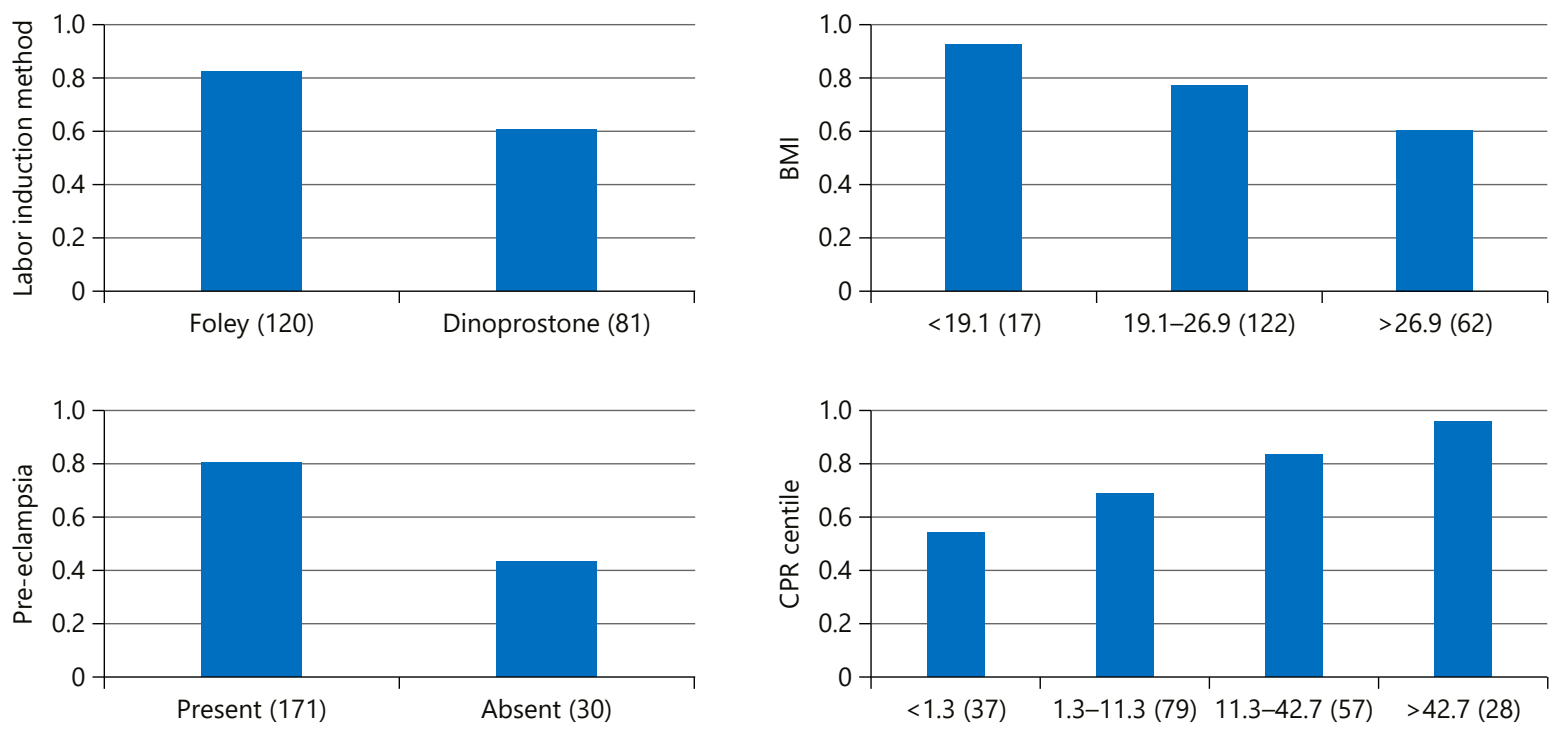

Fig. 2. Vaginal delivery probability according to the main predictive variables on univariate analysis. The cut-offs for splitting the continuous variables were automatically chosen by the decision trees. The number of cases in each subgroup is shown in parentheses. CPR, cerebro-placental ratio.

Fig. 3. Predictive model of vaginal delivery after labor induction in stage I FGR.

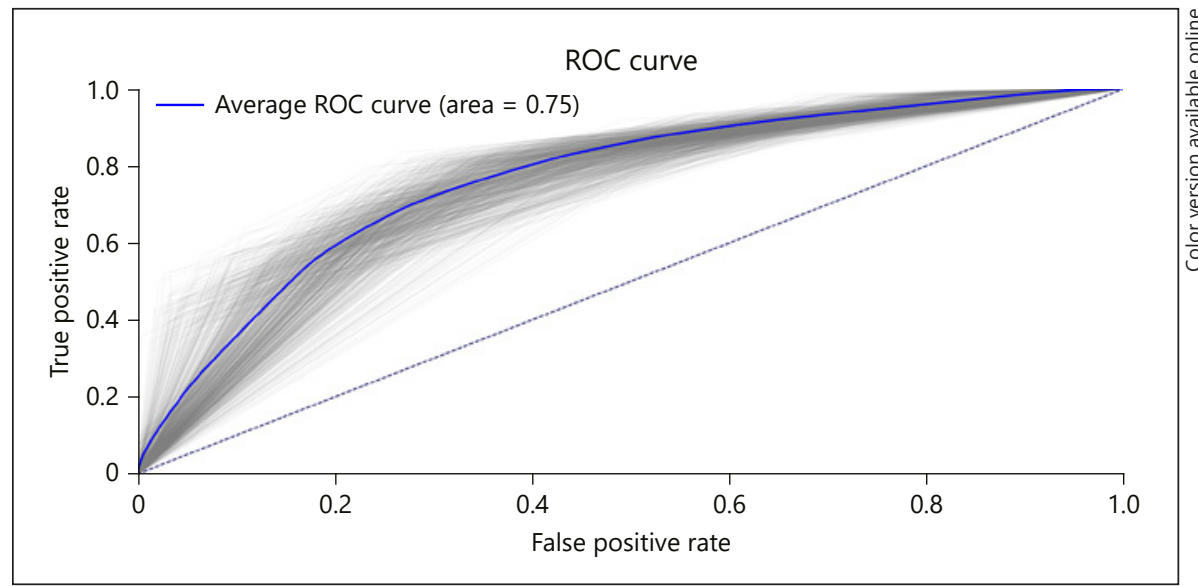

dictive variable is presented in Figure 2. The cut-offs for splitting the continuous variables were automatically chosen by the decision trees. The resulting multivariate model presented an AUC of 0.84 (95\% CI 0.79-0.90). Figure 3 presents the computed graph after bootstrapping with 1,000 re-samples with an AUC of 0.75 (95\% CI $0.70-$ 0.80 ). Table 4 shows 4 hypothetic cases that differ in the main predictive parameters (method of labor induction and CPR centile) in which the vaginal delivery model is applied.

Prognostic Factors of Labor Induction in FGR

\section{Discussion}

\section{Main Findings}

Our study shows that the chances of vaginal delivery after the induction of labor with cervical ripening in lateonset FGR at term are satisfactory (74.6\%). We have identified a series of factors that favor the success of vaginal delivery in late-onset FGR before labor induction is attempted: use of a Foley balloon as the labor induction method, higher CPR centiles, absence of pre-eclampsia, 
Table 4. Probability of vaginal delivery in 4 hypothetical examples in which there are changes in the labor induction method and fetal hemodynamic status

\begin{tabular}{lllll}
\hline Example & A & B & C & D \\
\hline Labor induction method & Foley & Foley & Dinoprostone & Dinoprostone \\
CPR centile & 45 & 3 & 45 & 3 \\
BMI & 23.5 & 23.5 & 23.5 & 23.5 \\
Pre-eclampsia & No & No & No & No \\
OR for vaginal delivery (95\% CI) & $15.77(7.01-35.45)$ & $6.12(3.24-11.53)$ & $3.62(1.84-7.12)$ & $1.41(0.78-2.55)$ \\
Probability of vaginal delivery, \% (95\% CI) & $94.04(87.42-97.26)$ & $85.94(76.43-92.02)$ & $78.35(64.79-87.68)$ & $58.51(43.82-71.83)$ \\
\hline
\end{tabular}

BMI, body mass index; CI, confidence interval; CPR cerebro-placental ratio; OR, odds ratio.

and lower pre-gestational BMI. Two of them (CPR and the method for cervical ripening) have not been described in the general population so they are specific for this population. Only one of them is modifiable (the method for labor induction), with the use of the Foley balloon preferable over vaginal dinoprostone.

\section{Interpretation of the Results}

The finding of lower vaginal delivery rates in women with pre-eclampsia or higher BMI is consistent with the results of the majority of labor induction studies [17]. However, after performing a multivariate analysis, we did not find an association with other related variables, such as maternal ethnicity, the presence of a prior CS [18], or nulliparity [19]. Nonetheless, these results could be limited by the sample size of this study. Particularly in the case of prior CS, we only had 17 women in our cohort and although we found lower percentages of vaginal delivery in the univariant study, this could not be confirmed later by the logistic regression model.

A low Bishop score before labor induction has been linked to a considerable reduction of the chances of achieving vaginal delivery both in low-risk pregnancies [20] and in those with late-onset FGR, with only a 52\% vaginal delivery rate in the latter if the Bishop score is $<2$ and prostaglandins are used [21]. Nonetheless, other authors have found percentages of vaginal delivery that are close to $85 \%$ [10]. These differences in the vaginal delivery rate may be explained mainly by the method used for cervical ripening and the fetal status at the beginning of labor induction. According to the results of our multivariate model, the CPR is the best surrogate marker of fetal wellbeing in late-onset small for gestational age cases prior to delivery [21]. This correlates well with a study performed on 210 small for gestational age fetuses undergoing labor induction with prostaglandins or oxytocin, in which the CS rates in fetuses with a CPR $<5$ th centile was of 58.3 versus $29.3 \%$ in the normal CPR group $(p<0.001)$ [22]. These results are comparable to ours, especially when using dinoprostone for cervical ripening. Tachysystole with fetal repercussion was more frequent in the vaginal dinoprostone group, similarly to those described by other groups [23]. However, uterine tachysystole can be lowered and vaginal delivery rates improved by using the Foley balloon since, in our experience, the better the fetal situation at the beginning of the induction and the less uterine stimulation provoked by the method used for cervical ripening, the higher the chances are for a vaginal delivery.

To illustrate these concepts, Table 4 presents the hypothetical cases of 4 women with a normal BMI (23.5) and without pre-eclampsia, in which the only varying parameters are the method of labor induction and fetal Doppler. It can be observed how in cases with late-onset FGR and mild hemodynamic alterations (cases $\mathrm{B}$ and $\mathrm{D}$ ), the chances of achieving vaginal delivery are higher using a Foley balloon instead of dinoprostone: $85.9 \%$ (95\% CI 76.4-92.0\%) versus 58.5\% (95\% CI 42.8-71.8\%), respectively. Therefore, a Foley balloon should be considered as the cervical ripening method of choice when attempting labor induction in cases with late-onset FGR, especially in those with lower CPR centiles. This is of importance since, currently, the Foley balloon is not the first-line recommended method for cervical ripening in late-onset FGR [6].

Achieving vaginal delivery is important because it is associated with a better maternal recovery, lower maternal morbidity, and earlier and longer lasting maternal-neonatal bonding. Women with a vaginal delivery also have earlier and more effective breastfeeding [24] which, according to the World Health Organization, entails a significant reduction in neonatal mortality when compared to its instau- 
ration $24 \mathrm{~h}$ after delivery [25]. This is of special concern in cases that will benefit most from breastfeeding, such as FGR [26]. The application of our model of estimating the success of vaginal delivery after labor induction might encourage most women whose pregnancy has reached the term period with a fetus with late-onset stage I FGR to undergo labor induction with a Foley balloon, even in unfavorable cervical conditions, given the high chances of success. On the other hand, the reduced group of women with a low a priori chance of vaginal delivery according to our model should be counseled carefully, weighing the risks and benefits of an induction. In these cases, we could spare the time and resources associated with labor induction and the consequences of an intrapartum CS. This is especially true for women with a prior CS, in whom the risks of an intrapartum CS surpass those of an elective surgery [27].

\section{Strengths and Limitations}

The main strength of our model is that it has been exclusively designed for FGR and not small for gestational age cases, which have been shown to have 2 different profiles on tolerance to labor induction and vaginal delivery [28]. Another advantage over other studies is that it solely evaluates pre-induction variables and not intrapartum ones [29].

Among the limitations are those associated with any retrospective study and a relatively small sample size. They include observation, information, selection, and confusion bias. Caution should be taken not to generalize our findings to other populations since our sample was mainly composed by Caucasian, Hispanic, and North African women. However, even considering these limitations, we must remark that all cases were carefully diagnosed and managed under the same protocol and by the same experts in fetal medicine during the study period, only changing the cervical ripening method, which minimized the impact of the first 3 potential biases. We performed a multivariant regression analysis in order to limit the potential impact of the confusion bias. Finally, giv- en that the study was performed at a single center, our data need external validation.

In conclusion, labor induction in late-onset FGR in the early-term period is a safe procedure in which the success rates increase with the use of mechanical methods, higher CPR centiles, lower pre-gestational BMI, and the absence of pre-eclampsia.

\section{Acknowledgements}

We would like to show our gratitude to Mr. Claudio Noguera for his invaluable help with the statistical analysis in this paper.

\section{Statement of Ethics}

The study was approved by our hospital's Ethics Committee, with the collection of informed consent not required due to its retrospective character and use of encoded and anonymized data.

\section{Disclosure Statement}

The authors have no conflicts of interest to declare.

\section{Funding Sources}

We acknowledge the support of the Spanish Ministry of Health through a grant of the "Fondo de Investigaciones Sanitarias-Instituto de Investigación Carlos III” (FIS grant PI13/02405).

\section{Author Contributions}

C.V., I.H., and A.G.: conceptualization. C.V. and M.S.Q.: data curation. C.V.: formal analysis. C.V., I.H., P.G.-A., M.S.Q., E.S., and E.G.-M.: investigation. C.V., I.H., and A.G.: methodology. I.H.: project administration. A.G.: supervision. C.V., I.H., and A.G.: validation. C.V.: writing - original draft. I.H. and A.G.: writing - review and editing.

\section{References}

1 Miranda JO, Ramalho C, Henriques-Coelho $\mathrm{T}$, Areias JC. Fetal programming as a predictor of adult health or disease: the need to reevaluate fetal heart function. Heart Fail Rev. 2017 Nov;22(6):861-77.

2 Gordijn SJ, Beune IM, Thilaganathan B, Papageorghiou A, Baschat AA, Baker PN, et al. Consensus definition of fetal growth restriction: a Delphi procedure. Ultrasound Obstet Gynecol. 2016 Sep;48(3):333-9.

Prognostic Factors of Labor Induction in FGR
3 Crovetto F, Triunfo S, Crispi F, RodriguezSureda V, Roma E, Dominguez C, et al. Firsttrimester screening with specific algorithms for early- and late-onset fetal growth restriction. Ultrasound Obstet Gynecol. 2016 Sep; 48(3):340-8.

4 Rabinovich A, Tsemach T, Novack L, Mazor M, Rafaeli-Yehudai T, Staretz-Chacham O, et al. Late preterm and early term: when to induce a growth restricted fetus? A populationbased study. J Matern Fetal Neonatal Med. 2018 Apr;31(7):926-32.
5 Boers KE, Vijgen SM, Bijlenga D, van der Post JA, Bekedam DJ, Kwee A, et al.; DIGITAT study group. Induction versus expectant monitoring for intrauterine growth restriction at term: randomised equivalence trial (DIGITAT). BMJ. 2010 Dec;341:c7087.

6 McCowan LM, Figueras F, Anderson NH. Evidence-based national guidelines for the management of suspected fetal growth restriction: comparison, consensus, and controversy. Am J Obstet Gynecol. 2018 Feb;218(2 2S):S85568. 
7 Dos Santos F, Drymiotou S, Antequera Martin A, Mol BW, Gale C, Devane D, et al. Development of a core outcome set for trials on induction of labour: an international multistakeholder Delphi study. BJOG. 2018 Dec; 125(13):1673-80

8 Osmundson S, Ou-Yang RJ, Grobman WA. Elective induction compared with expectant management in nulliparous women with an unfavorable cervix. Obstet Gynecol. 2011 Mar;117(3):583-7.

9 Little SE, Caughey AB. Induction of labor and cesarean: what is the true relationship? Clin Obstet Gynecol. 2015 Jun;58(2):269-81.

10 Bernardes TP, Broekhuijsen K, Koopmans CM, Boers KE, van Wyk L, Tajik P, et al. Caesarean section rates and adverse neonatal outcomes after induction of labour versus expectant management in women with an unripe cervix: a secondary analysis of the HYPITAT and DIGITAT trials. BJOG. 2016 Aug;123(9):1501-8.

11 Villalaín C, Herraiz I, Quezada MS, GómezArriaga PI, Simón E, Gómez-Montes E, et al. Labor induction on late-onset fetal growth restriction: Foley balloon vs. vaginal dinoprostone. Fetal Diagn Ther. 2019;46(1):67-74.

12 Figueras F, Gratacos E. Stage-based approach to the management of fetal growth restriction. Prenat Diagn. 2014 Jul;34(7):655-9.

13 Fox NS, Saltzman DH, Roman AS, Klauser CK, Moshier E, Rebarber A. Intravaginal misoprostol versus Foley catheter for labour induction: a meta-analysis. BJOG. 2011 May; 118(6):647-54.

14 FIGO Subcommittee on Standards on Perinatal Medicine. Guidelines of the use of fetal monitoring. Int J Gynaecol Obstet. 1987;25: 159.
15 Report of the national high blood pressure education program working group on high blood pressure in pregnancy. Am J Obstet Gynecol. 2000 Jul;183(1):S1-22.

16 Hastie T, Tibshirani R, Friedman JH. The elements of statistical learning: data mining, inference, and prediction. 2nd ed. New York: Springer; 2009. https://doi.org/10.1007/9780-387-84858-7.

17 Tolcher MC, Holbert MR, Weaver AL, McGree ME, Olson JE, El-Nashar SA, et al. Predicting cesarean delivery after induction of labor among nulliparous women at term. Obstet Gynecol. 2015 Nov;126(5):1059-68.

18 Oros D, Garcia-Simon R, Clemente J, Fabre E, Romero MA, Montañes A. Predictors of perinatal outcomes and economic costs for lateterm induction of labour. Taiwan J Obstet Gynecol. 2017 Jun;56(3):286-90.

19 Pevzner L, Rayburn WF, Rumney P, Wing DA. Factors predicting successful labor induction with dinoprostone and misoprostol vaginal inserts. Obstet Gynecol. 2009 Aug; 114(2 Pt 1):261-7.

20 Vrouenraets FP, Roumen FJ, Dehing CJ, van den Akker ES, Aarts MJ, Scheve EJ. Bishop score and risk of cesarean delivery after induction of labor in nulliparous women. $\mathrm{Ob}$ stet Gynecol. 2005 Apr;105(4):690-7.

21 Garcia-Simon R, Figueras F, Savchev S, Fabre E, Gratacos E, Oros D. Cervical condition and fetal cerebral Doppler as determinants of adverse perinatal outcome after labor induction for late-onset small-for-gestational-age fetuses. Ultrasound Obstet Gynecol. 2015 Dec; 46(6):713-7.

22 Cruz-Martínez R, Figueras F, Hernandez-Andrade E, Oros D, Gratacos E. Fetal brain Doppler to predict cesarean delivery for nonreassuring fetal status in term small-for-gestational-age fetuses. Obstet Gynecol. 2011 Mar; 117(3):618-26.
23 Jozwiak M, Oude Rengerink K, Benthem M, van Beek E, Dijksterhuis MG, de Graaf IM, et al.; PROBAAT Study Group. Foley catheter versus vaginal prostaglandin E2 gel for induction of labour at term (PROBAAT trial): an open-label, randomised controlled trial. Lancet. 2011 Dec;378(9809):2095-103.

24 Regan J, Thompson A, DeFranco E. The influence of mode of delivery on breastfeeding initiation in women with a prior cesarean delivery: a population-based study. Breastfeed Med. 2013 Apr;8(2):181-6.

25 World Health Organization. Guidelines on optimal feeding of low birth-weight infants in low-and middle-income countries. Geneva: WHO; 2011. pp. 16-45.

26 Tudehope D, Vento M, Bhutta Z, Pachi P. Nutritional requirements and feeding recommendations for small for gestational age infants. J Pediatr. 2013 Mar;162(3 Suppl):S81-9.

27 Hibbard JU, Ismail MA, Wang Y, Te C, Karrison T, Ismail MA. Failed vaginal birth after a cesarean section: how risky is it? I. Maternal morbidity. Am J Obstet Gynecol. 2001 Jun; 184(7):1365-71.

28 Figueras F, Savchev S, Triunfo S, Crovetto F, Gratacos E. An integrated model with classification criteria to predict small-for-gestational-age fetuses at risk of adverse perinatal outcome. Ultrasound Obstet Gynecol. 2015 Mar;45(3):279-85.

29 Kalafat E, Morales-Rosello J, Thilaganathan B, Tahera F, Khalil A. Risk of operative delivery for intrapartum fetal compromise in small-for-gestational-age fetuses at term: an internally validated prediction model. Am Obstet Gynecol. 2018 Jan;218(1):134.e1-8. 